\title{
SUMOylation alterations are associated with multidrug resistance in hepatocellular carcinoma
}

\author{
YU QIN ${ }^{1,2^{*}}$, HUIJING BAO ${ }^{3 *}$, YI PAN $^{4}$, MEILIN YIN $^{3}$, YUNDE LIU $^{3}$, SHANGWEI WU $^{3}$ and HUIKAI LI ${ }^{5}$ \\ ${ }^{1}$ Department of Diagnostics, College of Basic Medical Science, Tianjin Medical University, Tianjin 300070; \\ ${ }^{2}$ State Key Laboratory of Experimental Hematology, Institute of Hematology and Blood Diseases Hospital, \\ Chinese Academy of Medical Sciences and Peking Union Medical College, Tianjin 300020; \\ ${ }^{3}$ School of Laboratory Medicine, Tianjin Medical University, Tianjin 300203; \\ ${ }^{4}$ Key Laboratory of Cancer Prevention and Therapy; ${ }^{5}$ Department of Hepatobiliary Surgery, \\ Tianjin Medical University Cancer Institute and Hospital, Tianjin 300060, P.R. China
}

Received May 31, 2013; Accepted December 12, 2013

DOI: $10.3892 / \mathrm{mmr} .2014 .1882$

\begin{abstract}
The development of multidrug resistance (MDR) in hepatocellular carcinoma (HCC) may markedly reduce the efficacy of its chemotherapeutic treatment. However, the mechanism regulating the development of MDR in these tumors remains unknown. Given the emerging role of small ubiquitin-like modifier (SUMO)ylation in tumorigenesis, the possibility that it may also be involved in MDR development was investigated. The expression of SUMO-1 was first analyzed using immunohistochemistry in 20 cases of HCC. Nuclear SUMO-1 immunostaining was observed to be significantly increased in HCC specimens compared with matched adjacent non-neoplastic controls. To further investigate the potential role of SUMOylation in MDR in HCC, a multidrug-resistant HCC cell line, HepG2/R, was established by exposing HCC cells to gradually increasing concentrations of 5-fluorouracil. Western blot analysis revealed that the total levels of SUMO-1-conjugated proteins were markedly increased in HepG2/R cells compared with parental HepG2 cells. Furthermore, the expression of
\end{abstract}

Correspondence to: Dr Huikai Li, Department of Hepatobiliary Surgery, Tianjin Medical University Cancer Institute and Hospital, Huan-Hu-Xi Road, Ti-Yuan-Bei, Tianjin 300060, P.R. China E-mail:tjchlhk@sohu.com

Dr Shangwei Wu, School of Laboratory Medicine, Tianjin Medical University, No. 1 Guangdong Road, Tianjin 300203, P.R. China

E-mail: lab_wushangwei@kingmed.com.cn

*Contributed equally

Abbreviations: Aos1, activator of SUMO-1; HCC, hepatocellular carcinoma; MDR, multidrug resistance; SENP1, sentrin-specific protease 1; SUMO, small ubiquitin-like modifier; Uba2, ubiquitin-like modifier activating enzyme 2

Key words: SUMOylation, hepatocellular carcinoma, multidrug resistance, ubiquitin-like modifier activating enzyme 2, sentrin-specific protease 1 ubiquitin-like modifier activating enzyme 2 and sentrin-specific protease 1, important enzymes in the SUMOylation cascade, were markedly upregulated in the HepG2/R cell line. These findings support the hypothesis that SUMOylation is important in the development of MDR in HCC.

\section{Introduction}

Small ubiquitin-like modifier (SUMO)ylation is a post-translational modification that involves the covalent, but reversible, binding of SUMO (1). Four isoforms of SUMO have been identified, SUMO-1, $-2,-3$ and -4 . These proteins are $\sim 12 \mathrm{kDa}$ in size and are structurally similar to ubiquitin (2-5). Similar to ubiquitination, the SUMOylation cascade contains a SUMO-activating enzyme (E1), a SUMO-conjugating enzyme (E2) and different SUMO ligases (E3s). All SUMO isoforms are expressed as immature precursors with a variable C-terminus comprising 2-11 residues, following an essential GG motif. Maturation of SUMO involves the removal of this C-terminal tail by sentrin-specific proteases (SENPs) and exposing the diglycine motif required for conjugation. SUMO is then activated in an ATP-dependent manner by conjugation to the E1 heterodimer, activator of SUMO-1 (Aos1) and ubiquitin-like modifier activating enzyme 2 (Uba2). Activated SUMO is then transferred to the unique E2 enzyme Ubc9, which covalently attaches the modifier to the $\varepsilon$-amino group of a target lysine residue in the presence of an E3 SUMO ligase $(1,6,7)$. Cleavage after the diglycine motif by SENPs then removes the conjugated SUMO moieties from the target protein.

Due to its regulation of protein activity, post-translational modifications may have profound effects on carcinogenesis. SUMOylation has been shown to regulate various cellular pathways, including DNA replication and repair, chromosome packing and dynamics, genome integrity, nuclear transport, signal transduction and cell proliferation. These functions suggest that abnormal SUMOylation may affect cancer progression and metastasis (8-15).

Hepatocellular carcinoma (HCC) is the sixth most common type of malignancy worldwide. It is the fifth most 
common type of malignant disease in males, the eighth most common type of malignant disease in females and the third most common cause of cancer-related mortality following lung and stomach cancer (16). Systemic chemotherapy represents a palliative treatment following resection and/or transplant surgery for inoperable patients. However, intrinsic or acquired multidrug resistance (MDR) may interfere with the efficacy of chemotherapy in HCC. MDR, which results in increased drug efflux from cells, is associated with increased DNA repair and drug metabolism, as well as decreased apoptosis, all of which affect the tumor microenvironment (17-20). However, the role of SUMOylation in MDR or chemotherapy remains largely unknown.

The aim of this study was to analyze the resistance mechanisms of HCC. A novel multidrug resistant-HCC cell line (HepG2/R) was developed based on HepG2 cells, a model for chemosensitive HCC cells. We investigated the levels of SUMOylation in these cells and analyzed the specific role in the development of drug resistance in HCC cells.

\section{Materials and methods}

Chemotherapeutic drugs and antibodies. The chemotherapeutic drugs 5-fluorouracil (5-FU, catalog no. F6627-1G), paclitaxel (catalog no. O9512-5MG) and oxaliplatin (catalog no. O9512-5MG) were purchased from Sigma-Aldrich (St. Louis, MO, USA). Mouse antibodies against SUMO-1 (catalog no. sc-5308), Aos1 (catalog no. sc-271592), Uba2 (catalog no. sc-136359) and SENP1 (catalog no. sc-271360) were purchased from Santa Cruz Biotechnology, Inc. (Santa Cruz, CA, USA).

Cell lines. The HepG2 human HCC cell line was provided by Dr H Tang (Tianjin Life Science Research Center and Basic Medical School, Tianjin Medical University, Tianjin, China). The MDR sub-line HepG2/R was established by culturing HepG 2 cells in the presence of increasing concentrations of 5-FU $(0.1,0.2,0.4,0.8$ and $1.6 \mu \mathrm{g} / \mathrm{ml})$. HepG2/R cells were cultured in the presence of $1.6 \mu \mathrm{g} / \mathrm{ml}(12.3 \mathrm{mM}) 5-\mathrm{FU}$ to maintain their phenotype. All cells were cultured in Dulbecco's modified Eagle's medium supplemented with $10 \%$ fetal bovine serum, $100 \mu \mathrm{g} / \mathrm{ml}$ penicillin and $100 \mu \mathrm{g} / \mathrm{ml}$ streptomycin at $37^{\circ} \mathrm{C}$ in $5 \% \mathrm{CO}_{2}$.

Human samples. Samples of paraffin-embedded HCC tissues and their adjacent non-neoplastic controls $(n=20)$ were obtained from patients who underwent surgical resection at the Department of Hepatobiliary Surgery, Tianjin Medical University Cancer Institute and Hospital. HCC in these samples was confirmed by pathological diagnosis. This study was approved by the Committee for the Protection of Human Subjects of Tianjin Medical University Cancer Institute and Hospital and informed consent was obtained from all patients.

Immunohistochemical analysis. The expression of SUMO-1 was demonstrated by immunohistochemistry (IHC) according to the manufacturer's instructions (pv-6000; ZSGB-BIO, Beijing, China). Sections ( $4 \mu \mathrm{m}$-thick) were deparaffinized in xylene and endogenous peroxidase activity was blocked with $3 \%$ hydrogen peroxide in $50 \%$ methanol for $10 \mathrm{~min}$ at room temperature. The slides were rehydrated in a series of alcohol washes, and washed with phosphate-buffered saline (PBS) and pretreated with citrate buffer for $20 \mathrm{~min}$ at $95^{\circ} \mathrm{C}$. Following blocking of nonspecific binding sites with $10 \%$ normal goat serum in $\mathrm{PBS}$ for $20 \mathrm{~min}$ at $37^{\circ} \mathrm{C}$, the sections were incubated overnight at $4^{\circ} \mathrm{C}$ with anti-SUMO-1 (dilution, 1:200). Sections were rinsed with PBS and incubated with HRP-goat anti-mouse immunoglobulin $\mathrm{G}$ ( $\mathrm{IgG}$ ) for $20 \mathrm{~min}$ at $37^{\circ} \mathrm{C}$. Subsequently, the slides were incubated with 3,3'-diaminobenzidine chromogen for 5-10 min at room temperature and washed with distilled water. Finally, sections were counterstained with hematoxylin for $1 \mathrm{~min}$, dehydrated and mounted on coverslips. PBS without the primary antibody was used as a negative control.

Western blot analysis. Protein lysate was separated by SDS-PAGE and transferred onto polyvinylidene fluoride membranes (GE Healthcare, Piscataway, NJ, USA). Membranes were blocked in TBST (50 mM Tris $\mathrm{pH} \mathrm{7.5,}$ $0.15 \mathrm{M}$ sodium chloride, $0.5 \%$ Tween-20) containing $5 \%$ non-fat milk. Immunoblotting was performed using indicated antibodies diluted in TBST. The membranes were washed and incubated with goat anti-mouse IgG-horseradish peroxidase conjugate (Bio-Rad, Hercules, CA, USA). Specific complexes were visualized using an enhanced chemiluminescence detection system (GE Healthcare, Little Chalfont, UK).

3-(4,5-dimethylthiahiazol-2-yl)-2,5-diphenyl tetrazolium bromide (MTT) assay. MTT assays were used to evaluate rates of cell growth (Cell Growth Determination kit; Sigma-Aldrich, catalog no. CGD1). Cells were trypsinized into a single-cell suspension and seeded into 96-well plates at a density of $1 \times 10^{3}$ cells/well. At the indicated time points, $10 \%$ of the culture volume $(20 \mu \mathrm{l})$ MTT was added to each well and incubated for $4 \mathrm{~h}$ at $37^{\circ} \mathrm{C}$. Cultures were removed from the incubator and the resultant MTT formazan crystals were dissolved in $180 \mu \mathrm{l}$ MTT solvent $(0.1 \mathrm{~N} \mathrm{HCl}$ in anhydrous isopropanol). Absorbance values were measured using a microplate absorbance reader (Bio-Rad Laboratories, Richmond, CA, USA) at a wavelength of $570 \mathrm{~nm}$. To measure the effect of the chemotherapeutic agents on growth, the inhibitory rate was calculated as follows: Cell growth inhibition $(\%)=$ $\left[\mathrm{A}_{570}(\right.$ control $)-\mathrm{A}_{570}($ experiment $\left.)\right] / \mathrm{A}_{570}($ control $) \times 100$.

Statistical analysis. All experiments were performed at least in triplicate. Statistical significance was evaluated using Student's t-test. $\mathrm{P}<0.05$ was considered to indicate a statistically significant difference.

\section{Results}

Establishment and characterization of the HCC MDR cell line HepG2/R. To obtain multidrug resistant-cells, HepG2 cells were treated with increasing concentrations of 5-FU between 0.1 and $1.6 \mu \mathrm{g} / \mathrm{ml}$. The drug-resistant subclone HepG2/R was established almost one year following initiation of treatment. HepG2/R cells were morphologically distinct from their parental cell line in that they demonstrated increased pseudopodia formation (Fig. 1A). Cell proliferation did not 
A

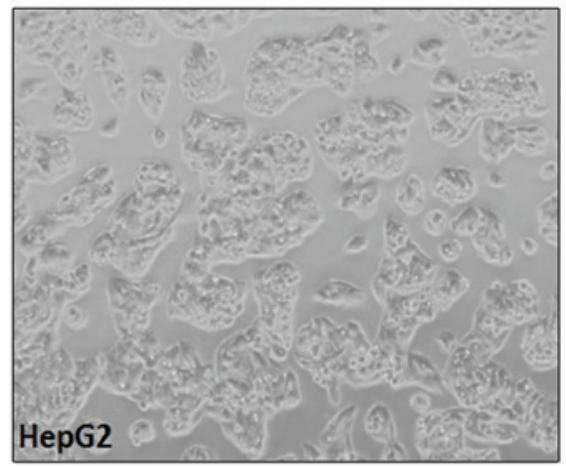

B

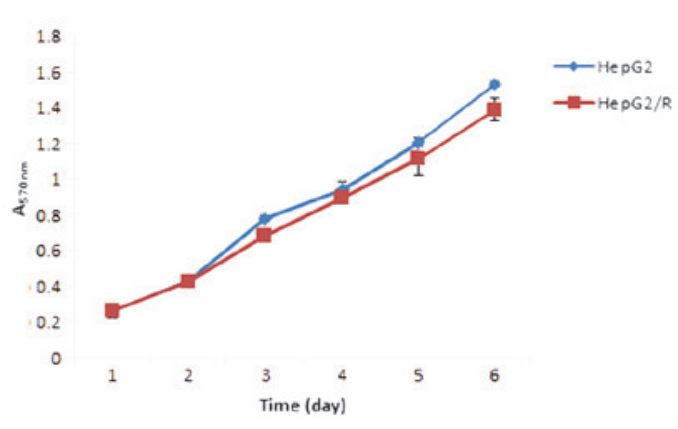

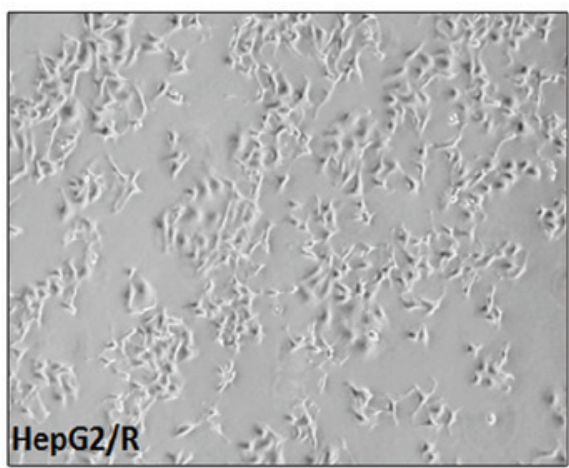

C

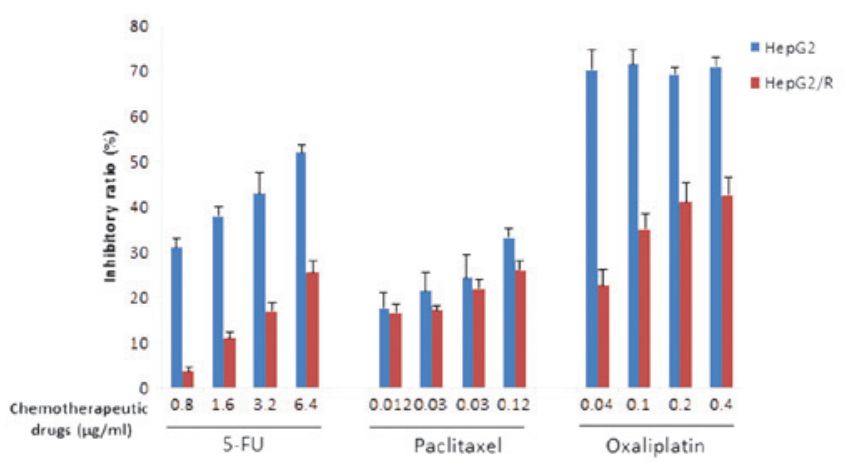

Figure 1. Characterization of the hepatocellular carcinoma multidrug resistance cell line, HepG2/R. (A) HepG2/R cells are morphologically distinct from their parental HepG2 cells, showing increased pseudopodia formation. (B) The MTT assay was used to measure cell growth over a six-day period. Proliferation rates of the HepG2 cells are similar to those of the HepG2/R cells. The results are expressed as the mean \pm SD of three assays. (C) Cells were treated with various concentrations of chemotherapeutic drugs, 5-FU, paclitaxel and oxaliplatin, and inhibitory ratios were measured using the MTT assay. Results are expressed as the mean \pm SD from three assays. SD, standard deviation; MTT, 3-(4,5-dimethylthiahiazol-2-yl)-2,5-diphenyl tetrazolium bromide; 5-FU, 5-fluorouracil. Magnification, $\mathrm{x} 200$.

A

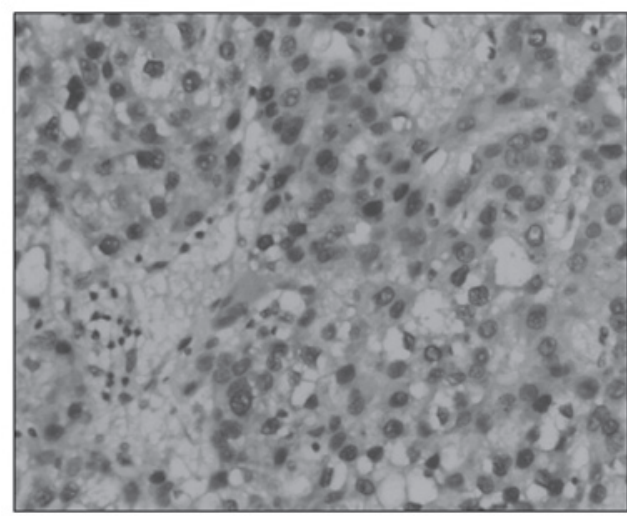

B

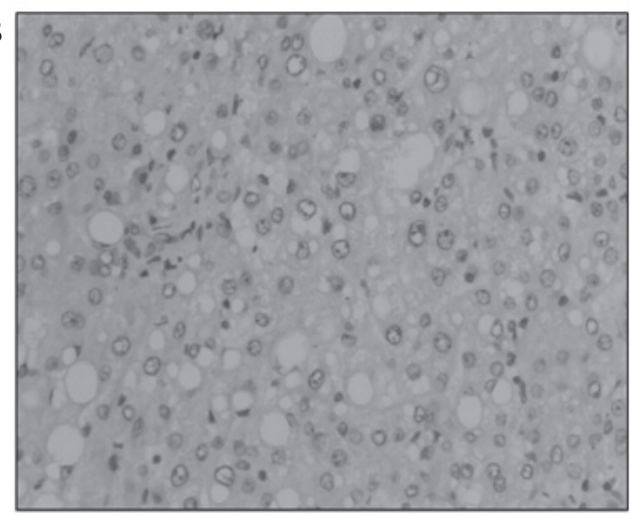

Figure 2. SUMO-1 immunostaining of HCC tissue and the adjacent non-neoplastic liver tissue (magnification, x200). (A) Strong nuclear SUMO-1 staining in HCC tissue. (B) Nuclear SUMO-1 staining was not detected in non-neoplastic liver tissue. Each staining experiment was performed with a negative control. SUMO-1, small ubiquitin-like modifier; HCC, hepatocellular carcinoma. Immunoperoxidase stain; magnification x200.

differ significantly between the HepG2 and HepG2/R cells (Fig. 1B). Next, the sensitivity of the two cell lines to specific concentrations of chemotherapeutic drugs was investigated. Following treatment with $0.8-6.4 \mu \mathrm{g} / \mathrm{ml} \mathrm{5-FU,} \mathrm{the} \mathrm{inhibitory}$ ratios of HepG2/R were markedly lower compared with those of the parental HepG2 cells (Fig. 1C). The cross-resistance to other chemotherapeutic drugs, including paclitaxel and oxaliplatin was also investigated. HepG2/R cells were observed to develop cross-resistance to paclitaxel and oxaliplatin to varying degrees (Fig. 1C).
SUMO-1 is overexpressed in clinical HCC samples. Immunostaining was used to evaluate the SUMO-1 expression in tumor cells from HCC tissues and normal cells from the adjacent non-neoplastic liver tissue. Nuclear SUMO-1 expression was detected in HCC neoplastic cells from all samples (100\%). However, in the matched non-neoplastic controls, little or no SUMO-1 expression was observed (Fig. 2A and B).

SUMO-1-conjugated proteins differ between HepG2 and $H e p G 2 / R$ cells. To investigate the pattern of SUMOylation 


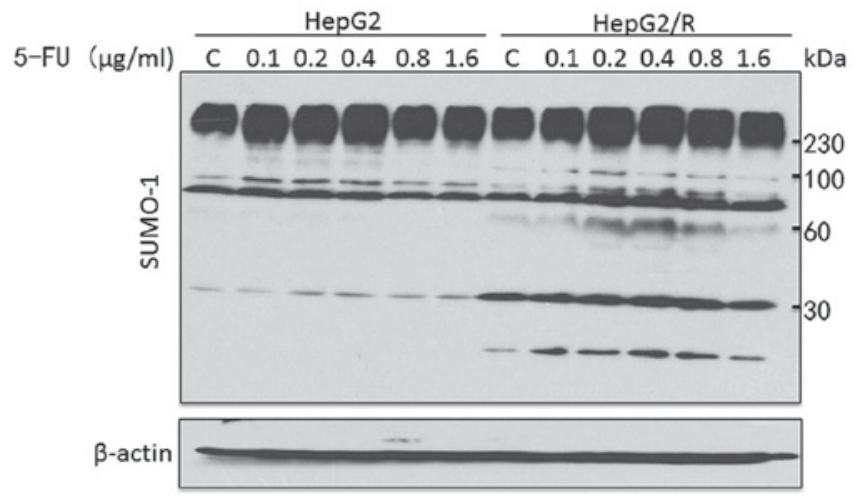

Figure 3. Total levels of SUMO-1-conjugated proteins are increased by different doses of 5-FU in HepG2/R compared with HepG2 cells. A distinct SUMO-1-conjugated protein $(60 \mathrm{kDa})$ and free SUMO-1 protein $(12 \mathrm{kDa})$ were detected in HepG2/R cells. Expression of SUMO-1-conjugated proteins at 30 and $100 \mathrm{kDa}$ were also clearly increased. 5-FU, 5-fluorouracil. SUMO-1, small ubiquitin-like modifier.

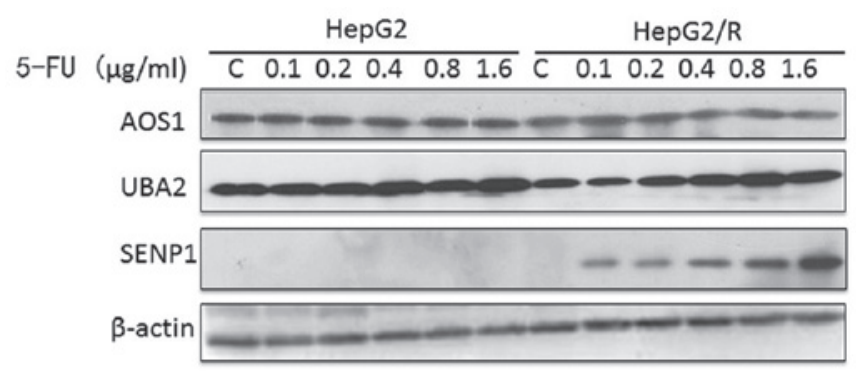

Figure 4. Expression levels of SUMOylation enzymes in HepG2 and HepG2/R cells treated with different doses of 5-FU. Aos1 expression was unaffected by 5 -FU treatment in $\mathrm{HepG} 2$ or HepG2/R cells. Uba2 levels were markedly increased in a dose-dependent manner in HepG2/R cells compared with HepG2 cells. SENP1 was not detected in HepG2 cells, but was expressed in a dose-dependent manner in HepG2/R cells. 5-FU, 5-fluorouracil; SUMO-1, small ubiquitin-like modifier; Aos1, activator of SUMO-1; Uba2, ubiquitin-like modifier activating enzyme 2; SENP1, sentrin-specific protease 1 .

in HepG2 and HepG2/R cells treated with 5-FU at different concentrations, whole-cell lysates were probed with a SUMO-1 antibody. The total levels of SUMOylation were significantly enhanced in HepG2/R cells. As shown in Fig. 3, distinct SUMO-1-conjugated and free SUMO-1 proteins were detected in HepG2/R cells at $\sim 60$ and $12 \mathrm{kDa}$, respectively. The levels of SUMO-1-conjugated proteins at 30 and $100 \mathrm{kDa}$ were also markedly increased.

Uba2 and SENP1 are upregulated in the HepG2/R cell line. In addition, the levels of SUMOylation enzymes in the HepG2 and HepG2/R cells were compared to determine whether the SUMOylation pathway differed. Although Aos1 levels were unaffected by the 5-FU treatment, Uba2 and SENP1 levels increased in a dose-dependent manner in HepG2/R cells (Fig. 4).

\section{Discussion}

Drug resistance is a major obstacle for the successful chemotherapeutic treatment of HCC. Often following chemotherapy, tumors fail to reduce in size or the cancer recurs following an initial response. MDR is particularly problematic as it involves the simultaneous resistance to numerous chemotherapeutic drugs of different classes. However, the mechanism of MDR remains unclear. To elucidate the molecular mechanisms in HCC a multidrug-resistant cell model is required. 5-FU has been used alone or in combination with other chemotherapeutic drugs for HCC and other malignant tumors $(21,22)$. Thus, an MDR HCC cell line, HepG2/R, was established by exposing HepG2 cells to increasing concentrations of 5-FU over one year. HepG2/R cells exhibited a strong resistance to 5-FU and also demonstrated cross-resistance to multiple, structurally diverse chemotherapeutic agents, including paclitaxel and oxaliplatin.

SUMOylation is essential for the maintenance of protein stability, transcriptional regulation and modification of particular transcription factors. SUMOylation is also involved in cellular processes, including mitosis, differentiation, senescence and apoptosis (8,23-26). Guo et al (27) reported that SUMO-1 was overexpressed in all HCC cell lines and clinical HCC samples that were tested. In agreement with this observation, the current study showed an increase in the expression of SUMO-1 in clinical HCC samples compared with the adjacent non-neoplastic liver tissue controls. This suggests that SUMOylation may be important in HCC. In addition, total levels of SUMO-1-conjugated proteins are increased in HepG2/R cells compared with parental HepG2 cells. This demonstrates that higher levels of SUMOylation are associated with MDR in HCC, possibly by affecting pathways that induce MDR.

SUMOylation is a dynamic process involving the conjugation (Aos1/Uba2, Ubc9 and different E3 ligases) and de-conjugation machinery (SENPs) (1). Notably, these enzymes have also been observed to affect tumorigenesis. Inactivation of Uba2 leads to mitotic catastrophe and cell death following $M y c$ hyperactivation. Uba2 inhibition switches a transcriptional subprogram of $M y c$ from activated to repressed (28). SENP1 is highly expressed in prostate cancer and correlates with hypoxia-inducing factor 1 (HIF-1) expression, which is associated with an increase in P-glycoprotein expression and the occurrence of MDR in tumor cells $(29,30)$. In the current study, high concentrations of 5-FU were found to increase Uba 2 expression in HCC MDR cells. This increase may affect cellular processes regulated by oncogenic Myc. 5-FU treatment also increased SENP1 expression in a dose-dependent manner, potentially implicating HIF-1 in the development of MDR in HCC.

In conclusion, the development of MDR in HCC was observed to be associated with increased SUMOylation, likely due to the increased expression of Uba2 and SENP1. These results suggest that the SUMOylation pathway may be a novel therapeutic target for preventing the development of MDR in $\mathrm{HCC}$, thus, increasing the efficacy of chemotherapy for this widespread disease.

\section{Acknowledgements}

This study was supported by scientific research grants from Tianjin Medical University (grant nos. 2010ky04 and 2011ky42). 


\section{References}

1. Geiss-Friedlander R and Melchior F: Concepts in sumoylation: a decade on. Nat Rev Mol Cell Biol 8: 947-956, 2007.

2. Meluh PB and Koshland D: Evidence that the MIF2 gene of Saccharomyces cerevisiae encodes a centromere protein with homology to the mammalian centromere protein CENP-C. Mol Biol Cell 6: 793-807, 1995.

3. Shen Z, Pardington-Purtymun PE, Comeaux JC, Moyzis RK and Chen DJ: UBL1, a human ubiquitin-like protein associating with human RAD51/RAD52 proteins. Genomics 36: 271-279, 1996.

4. Okura T, Gong L, Kamitani T, Wada T, Okura I, Wei CF, Chang HM and Yeh ET: Protection against Fas/APO-1- and tumor necrosis factor-mediated cell death by a novel protein, sentrin. J Immunol 157: 4277-4281, 1996.

5. Seeler JS and Dejean A: Nuclear and unclear functions of SUMO. Nat Rev Mol Cell Biol 4: 690-699, 2003.

6. Wadosky KM and Willis MS: The story so far: post-translational regulation of peroxisome proliferator-activated receptors by ubiquitination and SUMOylation. Am J Physiol Heart Circ Physiol 302: H515-H526, 2012

7. Gong L, Li B, Millas S and Yeh ET: Molecular cloning and characterization of human AOS1 and UBA2, components of the sentrin-activating enzyme complex. FEBS Lett 448: 185-189, 1999.

8. Muller S, Berger M, Lehembre F, Seeler JS, Haupt Y and Dejean A: c-Jun and p53 activity is modulated by SUMO-1 modification. J Biol Chem 275: 13321-13329, 2000.

9. Liu G and Warbrick E: The p66 and p12 subunits of DNA polymerase delta are modified by ubiquitin and ubiquitin-like proteins. Biochem Biophys Res Commun 349: 360-366, 2006.

10. Tanaka K, Nishide J, Okazaki K, Kato H, Niwa O, Nakagawa T, Matsuda H, Kawamukai M and Murakami Y: Characterization of a fission yeast SUMO-1 homologue, pmt3p, required for multiple nuclear events, including the control of telomere length and chromosome segregation. Mol Cell Biol 19: 8660-8672, 1999.

11. Bachant J, Alcasabas A, Blat Y, Kleckner N and Elledge SJ: The SUMO-1 isopeptidase Smt4 is linked to centromeric cohesion through SUMO-1 modification of DNA topoisomerase II. Mol Cell 9: 1169-1182, 2002.

12. Azuma Y, Arnaoutov A and Dasso M: SUMO-2/3 regulates topoisomerase II in mitosis. J Cell Biol 163: 477-487, 2003.

13. Wu N, Kong X, Ji Z, Zeng W, Potts PR, Yokomori K and Yu H: Scc1 sumoylation by Mms21 promotes sister chromatid recombination through counteracting Wapl. Genes Dev 26: 1473-1485, 2012.

14. McAleenan A, Cordon-Preciado V, Clemente-Blanco A, Liu IC, Sen N, Leonard J, Jarmuz A and Aragón L: SUMOylation of the $\alpha$-kleisin subunit of cohesin is required for DNA damage-induced cohesion. Curr Biol 22: 1564-1575, 2012.

15. Luo K, Zhang H, Wang L, Yuan J and Lou Z: Sumoylation of MDC1 is important for proper DNA damage response. EMBO J 31: 3008-3019, 2012.
16. Ferenci P, Fried M, Labrecque D, Bruix J, Sherman M, Omata M, Heathcote J, Piratsivuth T, Kew M, Otegbayo JA, et al: Hepatocellular carcinoma (HCC): a global perspective. J Clin Gastroenterol 44: 239-245, 2010.

17. Correia AL and Bissell MJ: The tumor microenvironment is a dominant force in multidrug resistance. Drug Resist Updat 15: 39-49, 2012.

18. Doublier S, Belisario DC, Polimeni M, Annaratone L, Riganti C, Allia E, Ghigo D, Bosia A and Sapino A: HIF-1 activation induces doxorubicin resistance in MCF73-D spheroids via P-glycoprotein expression: a potential model of the chemo-resistance of invasive micropapillary carcinoma of the breast. BMC Cancer 12: 4, 2012.

19. Brökers N, Le-Huu S, Vogel S, Hagos Y, Katschinski DM and Kleinschmidt M: Increased chemoresistance induced by inhibition of HIF-prolyl-hydroxylase domain enzymes. Cancer Sci 101: 129-136, 2010.

20. Fabregat I, Roncero C and Fernández M: Survival and apoptosis: a dysregulated balance in liver cancer. Liver Int 27: 155-162, 2007.

21. Homann N, Pauligk C, Luley K, et al: Pathological complete remission in patients with oesophagogastric cancer receiving preoperative 5-fluorouracil, oxaliplatin and docetaxel. Int J Cancer 130: 1706-1713, 2012.

22. Kitagawa K, Kawada K, Morita S, et al: Prospective evaluation of corrected QT intervals and arrhythmias after exposure to epirubicin, cyclophosphamide, and 5-fluorouracil in women with breast cancer. Ann Oncol 23: 743-747, 2012.

23. Nagai S, Davoodi N and Gasser SM: Nuclear organization in genome stability: SUMO connections. Cell Res 21: 474-485, 2011.

24. Cai Q and Robertson ES: Ubiquitin/SUMO modification regulates VHL protein stability and nucleocytoplasmic localization. PLoS One 5: e12636, 2010.

25. Ding B, Sun Y and Huang J: Overexpression of SKI oncoprotein leads to p53 degradation through regulation of MDM2 protein sumoylation. J Biol Chem 287: 14621-14630, 2012

26. Bettermann K, Benesch M, Weis S and Haybaeck J: SUMOylation in carcinogenesis. Cancer Lett 316: 113-125, 2012.

27. Guo WH, Yuan LH, Xiao ZH, Liu D and Zhang JX: Overexpression of SUMO-1 in hepatocellular carcinoma: a latent target for diagnosis and therapy of hepatoma. J Cancer Res Clin Oncol 137: 533-541, 2011.

28. Kessler JD, Kahle KT, Sun T, et al: A SUMOylation-dependent transcriptional subprogram is required for Myc-driven tumorigenesis. Science 335: 348-353, 2012

29. Wang Q, Xia N, Li T, et al: SUMO-specific protease 1 promotes prostate cancer progression and metastasis. Oncogene 32: 2493-2498, 2013

30. Wartenberg M, Ling FC, Muschen M, et al: Regulation of the multidrug resistance transporter P-glycoprotein in multicellular tumor spheroids by hypoxia-inducible factor (HIF-1) and reactive oxygen species. FASEB J 17: 503-505, 2003. 